Article

\title{
Combined Treatment with Doxorubicin and Rapamycin Is Effective against In Vitro and In Vivo Models of Human Glioblastoma
}

\author{
Anna Lisa Iorio ${ }^{1, *}$, Martina Da Ros ${ }^{1}{ }^{1}$, Claudio Pisano $^{2}$, Maurizio de Martino ${ }^{1}$, \\ Lorenzo Genitori ${ }^{1}$ and Iacopo Sardi ${ }^{1}$ (D) \\ 1 Neuro-oncology Unit, Department of Pediatric Oncology, Meyer Children's Hospital, 50139 Florence, Italy; \\ martina.daros@meyer.it (M.D.R.); maurizio.demartino@unifi.it (M.d.M); lorenzo.genitori@meyer.it (L.G.); \\ iacopo.sardi@meyer.it (I.S.) \\ 2 BIOGEM Research Institute, 83031 Ariano Irpino, Italy; claudio.pisano@biogem.it \\ * Correspondence: annalisa.iorio@meyer.it; Tel.: +39-055-5662631
}

Received: 11 February 2019; Accepted: 6 March 2019; Published: 9 March 2019

\begin{abstract}
Despite numerous clinical trials, glioblastoma (GBM) remains a tumor that is difficult to treat. The aim of this study was to investigate the potential of a new pharmacological approach, combining doxorubicin (Dox) and rapamycin (Rapa), in in vitro and in vivo GBM models. Cytotoxic and anti-proliferative effects of Rapa plus Dox treatments were analyzed in GBM cell lines. The in vivo effectiveness of these treatments was investigated in an orthotopic xenograft mice model of GBM. In vitro results demonstrated that prolonged exposure to Rapa sensitize GBM cells to Dox treatments. In vivo results demonstrated that Rapa $(5 \mathrm{mg} / \mathrm{kg}$ ) plus Dox $(5 \mathrm{mg} / \mathrm{kg})$ determined the major tumor growth inhibition ( $-97.29 \%$ vs. control) but results in greater toxicity. The combination Rapa plus Dox $(2.5 \mathrm{mg} / \mathrm{kg})$ showed a tumor inhibition like Rapa plus Dox $(5 \mathrm{mg} / \mathrm{kg})$ with a toxicity comparable to Rapa alone. Thus, this study demonstrated the efficacy of this pharmacological approach, providing the rationale for a clinical application of this combinational therapy in "poor-responder" GBM patients.
\end{abstract}

Keywords: doxorubicin; rapamycin; glioblastoma; mTOR inhibitor; chemotherapy

\section{Introduction}

Glioblastoma (GBM) is the most frequent and lethal brain tumor in adults. Location, aggressiveness, and diffuse infiltrative growth make GBM therapy extremely challenging and frequently unsuccessful. Consolidated first-line treatment of newly diagnosed GBM consists of maximal surgical resection, when possible, followed by radiotherapy (RT) and adjuvant temozolomide (TMZ) [1]. This therapy provides a median overall survival of 12 months, and a five-year survival rate of less than $5 \%[2,3]$. Such a poor prognosis generates a compelling need for innovative and effective therapeutic strategies.

Doxorubicin (Dox) is an anthracycline antibiotic with antineoplastic activity. This historical drug is often used, together with other chemotherapy agents, in many tumors. Different groups have reported the effectiveness of Dox also against GBM, both in preclinical models. Veringa et al. have demonstrated that Dox showed a high cytotoxic effect on primary GBM cell lines, more than conventional used TMZ and etoposide [4].

Our group have also recently investigated the efficacy of Dox treatment against GBM, reporting how Dox, in combination with other drugs, has a high cytotoxic effect against GBM cell lines and determined a decrease in volume by an orthotopic xenograft mice model of human GBM $[5,6]$. 
Cancer development is regulated by numerous and complex mechanisms. Hyperactivation of downstream phosphatidylinositol 3-kinase (PI3K)/AKT/ mammalian target of rapamycin (mTOR) pathway is a common occurrence of human glioma $[7,8]$.

$\mathrm{mTOR}$ is a Ser/Thr protein kinase that plays a critical role in cellular responses to growth factors and stress, controlling mRNA translation, ribosome biogenesis, autophagy, and metabolism [9]. Rapa, also known as Sirolimus, is a clinically approved immunosuppressant that has shown promising antitumor activity. Rapa, a specific inhibitor of mTOR function, blocks serum-stimulated Ser-2448 phosphorylation by AKT and other downstream effectors, such as p70S6 kinase [10]. This drug has been shown to be effective against intracerebral glioma xenografts and has a cytostatic effect against gliomas [11]; several Rapa derivatives (rapalogues) have been synthesized, namely temsirolimus, everolimus, and ridaforolimus. Currently, more than 30 clinical trials involve Rapa or its derivatives in brain and Central Nervous System (CNS) cancer therapy.

Basing on these evidences, the purpose of our study was to investigate the in vitro and in vivo efficacy of the co-treatment Rapa plus Dox, in order to elucidate the potential of this combined treatment as available approach for GBM therapy.

\section{Materials and Methods}

\subsection{MTT Assay}

The effect of the treatments, on tumor cells growth, was measured using the MTT Cell Proliferation Assay (Cayman Chemical, Ann Arbor, MI, USA), following the manufacturer's instructions.

Three human GBM cell lines, A172, U87MG, and T98G, were employed in this study (American Type Culture Collection (ATCC), Manassas, VA, USA). U87MG and T98G were grown in Eagle's Minimum Essential Medium, while A172 was grown in Dulbecco's Modified Eagle Medium. Each medium was supplemented with $10 \%$ fetal bovine serum and $1 \%$ penicillin-streptomycin. All cell lines were maintained in a humidified atmosphere of $5 \% \mathrm{CO}_{2}-95 \%$ air at $37^{\circ} \mathrm{C}$.

GBM cell lines were seeded at the following densities: A172 and T98G $3 \times 10^{3}$ cells/well, U87MG, $5 \times 10^{3}$ cells/well in 96-well plates. After $24 \mathrm{~h}$, cells were treated with Rapa (10 nM, $100 \mathrm{nM}$, and $1 \mu \mathrm{M})$, Dox $(0.8 \mu \mathrm{M})$, and their combinations for $24 \mathrm{~h}, 48 \mathrm{~h}$, and $72 \mathrm{~h}$.

Optical density (OD) of each well was measured on a MULTISKAN FC (Thermo Scientific, Waltham, MA, USA) microplate reader at a test wavelength of $550 \mathrm{~nm}$. All experiments were performed three times in triplicate.

\subsection{Intracellular Dox Accumulation}

Dox cellular uptake was analyzed using a FACScan flow cytometer (Becton Dickinson, Mountain View, CA, USA), equipped with a $488 \mathrm{~nm}$ argon laser. The exponentially growing P-glycoprotein (P-gp) transfected MDCKII cells were treated with Rapa (10 nM, $100 \mathrm{nM}$, and $1 \mu \mathrm{M})$, Dox $(0.8 \mu \mathrm{M})$, and their combinations for $2 \mathrm{~h}$, according to the previously described method [6]. Dox fluorescence was measured at a flow rate of 8000 events/s. All experiments were performed two times in triplicate.

\subsection{Immunoblotting}

After washing cells with PBS 1X, monolayers of A172, U87MG and T98G cells were lysed in ice-cold RIPA buffer added with protease inhibitor cocktail (Sigma Aldrich, Saint Louis, MO, USA) and centrifuged at $4{ }^{\circ} \mathrm{C}$ for $10 \mathrm{~min}$ at $10,000 \times g$. The protein content was determined by using a BCA protein assay kit (Thermo Scientific, Waltham, MA, USA).

SDS-polyacrylamide gel electrophoresis was performed using $6 \%$ and $4 \%$ acrylamide for the separating and stacking gel, respectively. Blots were blocked with blocker milk 5\% solution and incubated overnight at $4{ }^{\circ} \mathrm{C}$ with anti-mTOR, anti-(P)ser2448mTOR (phosphorylation on 2448 is a biomarker of mTOR activity), and anti-vinculin primary antibodies (Cell Signaling Technology, Danvers, MA, USA). Blots were further incubated with secondary antibodies conjugated with 
horseradish peroxidase (Bio Rad, Hercules, CA, USA) for $1 \mathrm{~h}$ at room temperature. Proteins revelation were performed on chemi-doc imaging system (Bio Rad, Hercules, CA, USA) and densitometric analysis were made using ImageJ software.

\subsection{Animals}

110 Foxn 1 female nude mice were used in this study. Animals procedure of accommodation and care have been described in detail previously [6].

Briefly, mice were housed inside cages of polisulfone (4 mice/cage) with stainless steel cover-feed and sterilized and dust-free bedding cobs. Food and bedding were sterilized. Drinking water was supplied ad libitum and each mouse was daily offered a complete pellet diet (GLP 4RF21, Mucedola) throughout the study.

Biogem laboratories obtained the authorization for animal manipulation by the Italian Health Authority.

The Care and Husbandry of animals are in accordance with European Directives No. 2010/63 and with the Italian Regulatory system (D.L. vo No. 26, 4 March 2014). The official Biogem veterinarian approves all parts of this study concerning animal care.

Animals were inspected every day for mortality. Physical appearance, behavior, general and local clinical signs were also observed; all efforts were made to minimize the mice's suffering and mice showing clinical signs of pain and distress were euthanized for humane reasons by $\mathrm{CO}_{2}$ inhalation. At the end of experiments, all mice were euthanized by $\mathrm{CO}_{2}$ inhalation.

\subsection{Drugs and Reagents}

Dox (Pfizer) was diluted with sterile Saline solution, at different concentrations, and administered intra vein (tail vein, IV) in a volume of $5 \mathrm{~mL} / \mathrm{kg}$. Rapa (AdooQ Bioscience, Irvine, CA, USA) $5 \mathrm{mg} / \mathrm{kg}$ was dissolved in a solution containing 2\% DMSO, 30\% PEG400, 5\% Tween 80 , and 63\% MilliQ water and intraperitoneally (IP) administered in a volume of $5 \mathrm{mg} / \mathrm{kg}$. All chemicals and solvents were of the highest purity available from commercial sources and used without further purification.

\subsection{Experimental Design}

The procedure of tumor implantation has been described in detail previously [6].

Briefly, U87MG-luc2 (PerkinElmer Italia S.P.A., Monza, Italy), a human GBM cell line stably transfected with firefly luciferase gene (luc2), was used to establish the orthotopic glioma model. On the day of the tumor implantation, mice were micro-injected with $3 \times 10^{5}$ U87MG-luc2 cells in the left lobe of brain with infusion of $1 \mu \mathrm{L} / \mathrm{min}$ (with Hamilton syringe). Following intracranial tumor injection, bioluminescence (BLI) acquisitions were performed, at day 0,3 , and 7 , for baseline data. After tumor implantation (day +7$)$, mice were sorted based on BLI Average Radiance and randomly allocated as follows:

- Group 1 (Ctr): naïve mice received physiological solution $(5 \mathrm{~mL} / \mathrm{kg})$.

- $\quad$ Group 2 (Rapa $5 \mathrm{mg} / \mathrm{kg}$ ): daily administrations from day 7 to 42 .

- Group 3 (Dox $5 \mathrm{mg} / \mathrm{kg}$ ): weekly administrations (days 7, 14, 21, 28, and 35).

- Group 4 (Dox $2.5 \mathrm{mg} / \mathrm{kg}$ ): weekly administrations (days 7, 14, 21, 28, and 35).

- Group 5 (Dox $5 \mathrm{mg} / \mathrm{kg}+$ Rapa): weekly administrations of Dox and daily administrations of Rapa.

- Group 6 (Dox $2.5 \mathrm{mg} / \mathrm{kg}+\mathrm{Rapa}$ ): weekly administrations of Dox and daily administrations of Rapa.

Each experimental group contained 16 animals. Tumor growth and weight were carried out weekly and biweekly, respectively, for five weeks. 


\subsection{In Vivo Imaging}

Tumor growth and response to the treatments were monitored by BLI acquisitions, using IVIS 200 Spectrum Imaging System (PerkinElmer, Waltham, MA, USA). BLI acquisitions were performed immediately after tumor cells injection (day 0 and +7 ) and then weekly until the end of experiment.

In detail, mice were IP administered with $10 \mathrm{mg} / \mathrm{kg} / 10 \mathrm{~mL}$ of D-Luciferin (PerkinElmer (Waltham, MA, USA) Xenolight D-Luciferin Potassium Salt) and after $30 \mathrm{~min}$ were anesthetized by gas anesthesia ( $2 \%$ isoflurane).

All animals were placed into the IVIS 200 Imaging System to be imaged. BLI was expressed as average radiance in photons per $\mathrm{s} / \mathrm{cm}^{2}$ per steradian.

\subsection{Body Weight}

Body weight loss percent (\% BWL) was calculated as follows: 100 - (mean BW day-x/mean BW day- $1 \times 100$ ). Measurements were carried out biweekly and, as humane endpoint, animals showing a $\mathrm{BWL} \geq 15 \%$ were euthanized by $\mathrm{CO}_{2}$ inhalation.

\subsection{Histological Evaluation}

At day +42 , brains of 48 mice ( 8 animals / group) were collected, weighed, and preserved in $10 \%$ neutral buffered formalin solution (Sigma Aldrich, Saint Louis, MI, USA), in order to accomplish morphological studies. Based on BLI signals, the most representative five brains of each group were selected and submitted to the internal standard trimming, wax embedding, and cutting procedures. Serial slides were stained with haematoxylin-eosin or Ki67 (Dako, Santa Clara, CA, USA) immunostaining, in order to calculate the proliferation index within the tumor mass.

\subsection{Proliferation Index - Ki67 Quantitative Analysis}

Among the 30 embedded brains, the most representative specimens, at least three for each group, were considered for the proliferation index analysis.

In order to intercept tumor mass, samples were explored at different cutting levels; sections were collected and examined at regular intervals of $50 \mu \mathrm{m}$.

Ki67 immunodetection was performed on tumor-bearing specimens, identified on haematoxilyneosin stained slides; single or multiple sections were subjected to Ki67 immunohistochemistry, in order to reach the required number of nuclei.

Ki67 immunohistochemistry was performed by primary anti-human Ki67 mouse monoclonal antibody (Dako 1:100), incubated for $1 \mathrm{~h}$ at room temperature in antibody diluent (Leica Biosystems, Wetzlar, Germany, EU). The immunodetection technique was conducted with NovoLink Polymer Detection Systems kit (Leica Biosystems, Wetzlar, Germany, EU); Rodent Block M (Biocare Medical, Pacheco, CA, USA) was used for blocking endogenous mouse IgG and non-specific binding sites. Negative control (primary antibody omitted) was also performed.

Ki67 quantitative analysis was performed in representative fields of tumor mass, acquired at final magnification of $400 \times ; 1000$ tumor nuclei/case were counted. Proliferation index was expressed as the percentage of Ki67-positive tumor nuclei over the total number of neoplastic nuclei. For each case, the mean value was assigned as individual data; cell positivity was evaluated with TMarker software v2.146 version, a free tool for histopathological cell counting and staining estimation.

\subsection{Statistical Analysis}

Data were expressed as mean $\pm \mathrm{SD}$. Statistical analyses were performed using T-test between two groups while comparison between multiple groups were performed using 1- or 2-way ANOVA followed by post hoc corrections, as appropriate.

For the evaluation of in vivo results, outliers were removed through ROUT test $195 \%$ confidence interval) and the statistical analysis was performed using the unpaired Mann-Whitney U-test. 
For histological analysis, outliers were removed through ROUT test (95\% confidence interval), and statistical analyses were performed with the unpaired Mann-Whitney U-test or ANOVA followed by Tukey correction, as appropriate. All analyses were done using GraphPad Prism 5 and $p<0.05$ was considered statistically significant.

\section{Results}

\subsection{Cytotoxic Effect of Combined Treatments Rapa Plus Dox in GBM Cell Lines}

To assess the cytotoxic effects of the treatments, three GBM cell lines (A172, U87MG, and T98G) were exposed to Rapa (10 nM, $100 \mathrm{nM}$, and $1 \mu \mathrm{M})$, Dox $(0.8 \mu \mathrm{M})$, and their combinations, at different time points (Figure 1).
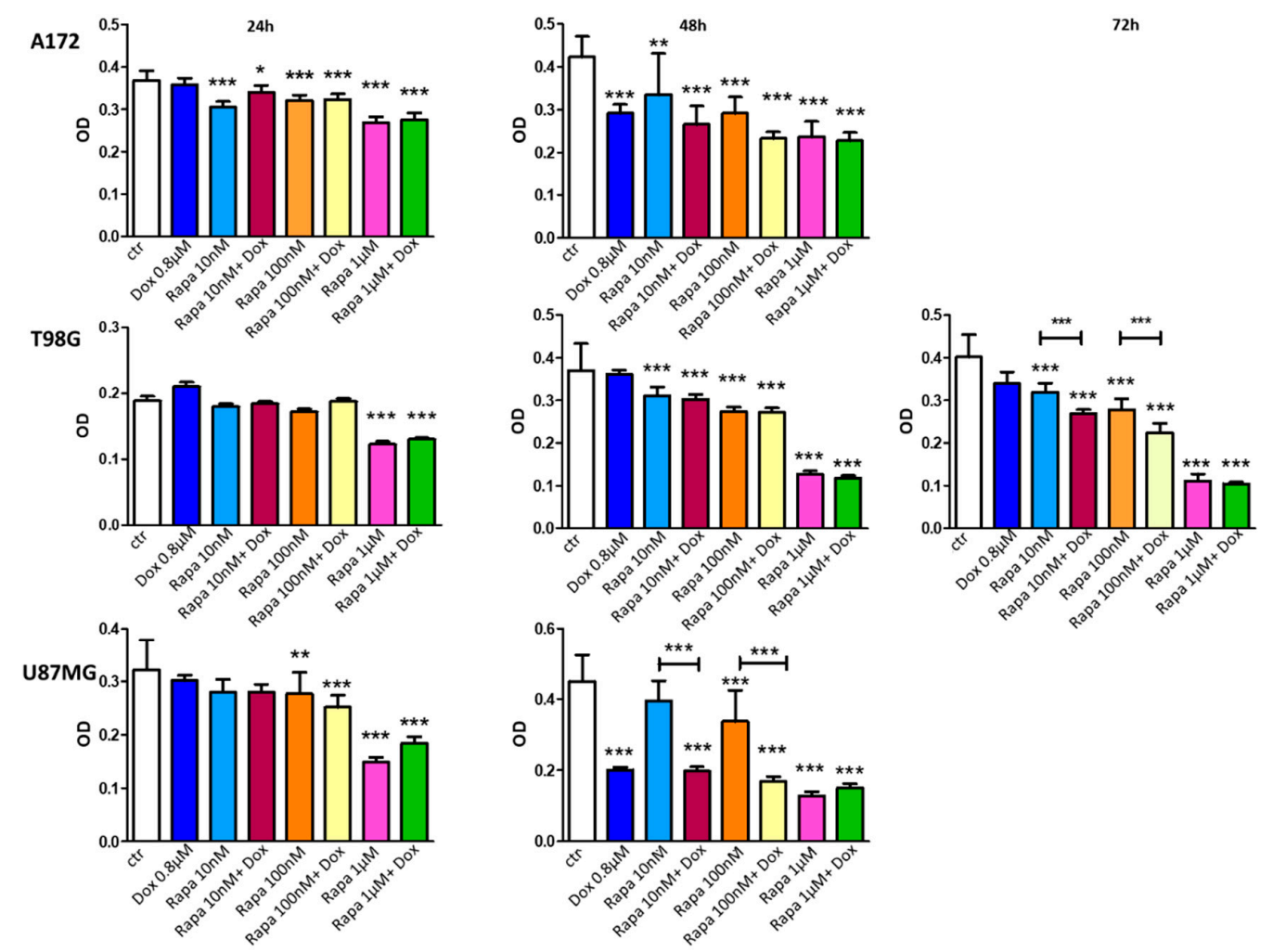

Figure 1. Effect of Dox, Rapa and their combinations on human glioblastoma (GBM) cell growth. U87MG, A172, and T98G cells were treated with various concentrations of drugs for $24 \mathrm{~h}$ and $48 \mathrm{~h}$. In T98G Dox resistant cells, the effects of Dox, Rapa, and their combinations have been also evaluated after $72 \mathrm{~h}$ of treatment. Statistical analysis was performed with 1-way ANOVA followed by Bonferroni post hoc correction ${ }^{*} p<0.05 ;{ }^{* *} p<0.01 ;{ }^{* * *} p<0.001$; ${ }^{* * *} p<0.0001$ vs. control group). Data were expressed as mean $\pm \mathrm{SD}$. This experiment was performed three times in triplicate.

Results obtained in A172 cells, after 24 h of treatments: Dox has no effect compared to control. All treatment including Rapa showed significant statistical differences vs. control. In conclusion, these data reported that the cytotoxic effect was related only to Rapa.

Results obtained in A172 cells, after 48 h of treatments: all treatments have significant effects vs. control. Comparing Rapa and Rapa plus Dox treatments, no significant differences were reported but we can observe a clear incremented cytotoxic effect in co-treated groups compared to Rapa and Dox single treatments.

Results obtained in T98G cells, after $24 \mathrm{~h}$ of treatments: all treatments have no effect vs. control, excluding Rapa $1 \mu \mathrm{M}$ and Rapa $1 \mu \mathrm{M}$ plus Dox $(0.1231 \pm 0.01356 ; 0.1304 \pm 0.007091$ vs. $0.1889 \pm 0.01972$, *** $p<0.001)$. No statistically significant differences were observed comparing between these two groups, indicating that the cytotoxic effect of the treatments was related only to Rapa. 
Results obtained in T98G cells, after $48 \mathrm{~h}$ of treatments: as previously reported [5], these cells remained resistant to Dox treatment while all other treatments showed significant effects vs. control. No significant differences were observed comparing between Rapa and Rapa plus Dox groups, at all concentrations, confirming that the cytotoxic effect was related only to Rapa.

Results obtained in T98G cells, after $72 \mathrm{~h}$ of treatments: once again, cells remained resistant to Dox treatment while all other treatments showed significant effects vs. control. Moreover, statistically significant results were obtained comparing between Rapa $10 \mathrm{nM}$ and Rapa $100 \mathrm{nM}$ vs. Rapa plus Dox treatments $\left(0.2691 \pm 0.009347\right.$ and $0.2244 \pm 0.02207$ vs. $0.3399 \pm 0.02708$ respectively; $\left.{ }^{* * * *} p<0.0001\right)$. OD value of Rapa $1 \mu \mathrm{M}$ plus Dox treatment was lower than Rapa $1 \mu \mathrm{M}$ alone but the difference was not statistically significant (Figure 1). Taken together, these results indicated that prolonged exposure to Rapa probably sensitizes T98G to Dox-mediated cytotoxic effect.

Results obtained in U87MG cells, after $24 \mathrm{~h}$ of treatments: Dox has no effect vs. control. Rapa alone and in combination with Dox showed significant statistical differences vs. control only at higher concentrations but no significant statistical differences were reported comparing Rapa alone vs. Rapa plus Dox, at all concentrations. As observed for A172, at $24 \mathrm{~h}$ of treatment, the cytotoxic effect was related only to Rapa.

Results obtained in U87MG cells, after $48 \mathrm{~h}$ of treatments: Dox alone has a marked significant effect vs. control $(0.2009 \pm 0.007373$ vs. $0.4487 \pm 0.07439$, *** $p<0.001)$. Rapa $10 \mathrm{nM}$ remained ineffective vs. control while Rapa $100 \mathrm{nM}$ and $1 \mu \mathrm{M}$ effects resulted in slight improvement compared to $24 \mathrm{~h}$. Significant statistical differences can be observed in all co-treated groups vs. control. Moreover, significant statistical differences were observed comparing Rapa 10 and $100 \mathrm{nM}$ vs. respective Rapa plus Dox groups (*** $p<0.001$ ) but, no difference were revealed comparing Dox vs. Rapa plus Dox combinations. Opposite to $24 \mathrm{~h}, 48 \mathrm{~h}$ data reported that Dox was majorly responsible for the cytotoxic effect of the treatments.

\subsection{Effect of Rapa Treatments on Dox Uptake}

Drug passage through blood brain barrier (BBB) is regulated by the presence of efflux transporters as P-gp. To determinate if Rapa treatments were able to influence Dox passage across cell membranes, we have investigated the cellular uptake of the anthracycline by using MDCKII cell line, a largely approved in vitro model of BBB.

Dox is an autoflorescent molecule and its intracellular accumulation is highly influenced by the presence of the P-gp protein [6], for this reason, we utilized MDCKII- P-gp transfected cells for our experiments. As reported in Figure 2A,B, no statistical differences were observed comparing Dox vs. Dox plus Rapa treated samples.

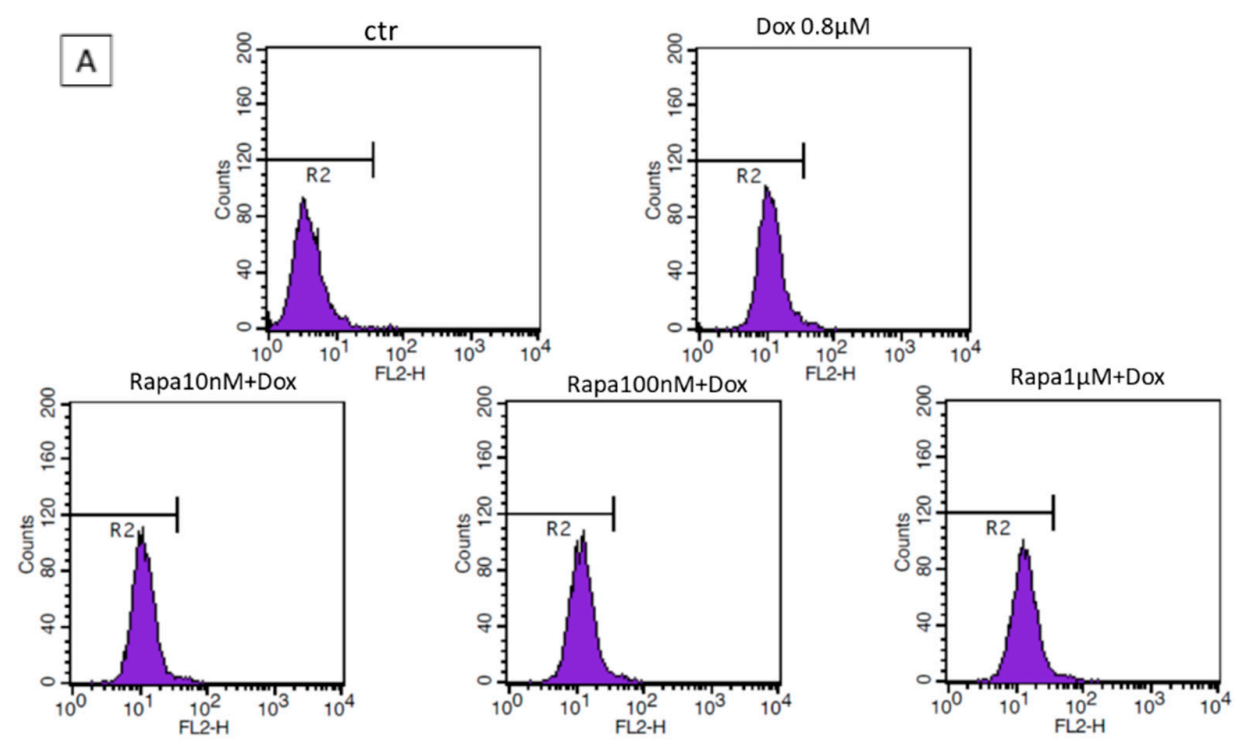

Figure 2. Cont. 


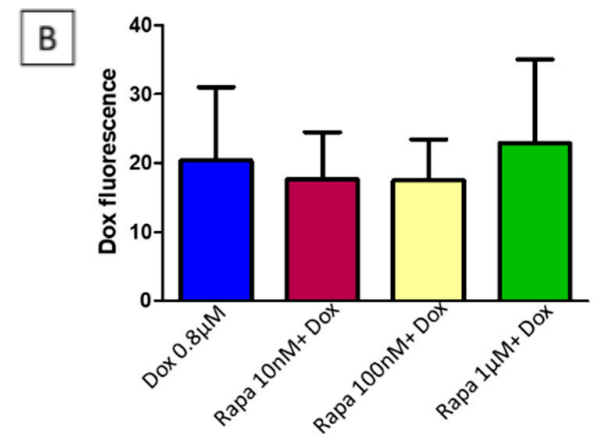

Figure 2. Effect of Rapa on Dox uptake in MDCKII P-gp transfected cells. (A) Cellular uptake of Dox by flow cytometry. Each peak represents the mean fluorescence intensity of Dox $(0.8 \mu \mathrm{M})$ in control and treated samples; (B) No significant difference can be observed comparing Dox fluorescence signals between cells treated with Dox $(0.8 \mu \mathrm{M})$ vs. Rapa plus Dox. Statistical analysis was performed by 1 -way ANOVA followed by Bonferroni post hoc correction. Data were expressed as mean $\pm \mathrm{SD}$. This experiment was performed two times in triplicate.

This data showed that Rapa, at all tested concentrations, not influence P-pg efflux activity and subsequently Dox uptake in MDCKII P-gp transfected cells. However, an incremented Dox cell accumulation can be appreciated in the presence of the higher Rapa concentration.

\subsection{Effect of Dox plus Rapa Treatments on mTOR Expression}

Immunoblotting results on U87 MG and A172 (a Dox sensible GBM cell lines) demonstrated that Dox has no effect on mTOR activity (phosphorylation of Ser2448) (Figure 3A). Meanwhile, as expected, Rapa treatments were able to reduce the phosphorylation of Ser2448, in both cell lines (Figure 3A). The most interesting results were obtained analyzing the effect of the combined treatments. Densitometric evaluation showed that Rapa 10 and Rapa 100 plus Dox reduced the activity of mTOR (U87MG:78\% and $43 \%$ phosphorylation of Ser2448 vs. control; A172:41\% and 58\% phosphorylation of Ser2448 vs. control) while, Rapa 1 plus Dox displayed a substantially inhibition of the kinase activity (U87MG: 28\% phosphorylation of Ser2448 vs. control; A172: 14\% phosphorylation of Ser2448 vs. control) (Figure 3B).

Densitometric evaluation of mTOR activation in T98G (a Dox resistant GBM cells) revealed that treatment with Dox alone increment phosphorylation of Ser2448 (132\% vs. control), while its combination with Rapa induced a $\sim 50 \%$ reduction of mTOR activation (Figure $3 \mathrm{~B}$ ).

\subsection{Effect of Dox Plus Rapa Treatments on Glioblastoma Growth in Mouse Xenograft Model}

One week after tumor implantation, 96 animals were divided into six groups and treated following the experimental schedule as described in Materials and Methods section. Treatments time-course and BLI evaluation of tumor growth are reported in Figure 4A.

Statistically significant results were obtained comparing treated groups vs. control group, at day +42 .

At day +42 the BLI Average Radiance of control group was $4.25 \mathrm{E}+06$ vs. $2.48 \mathrm{E}+05$ of Rapa group $\left({ }^{* *} p<0.01\right), 4.07 \mathrm{E}+05$ of Dox $5 \mathrm{mg} / \mathrm{kg}$ group $\left({ }^{* *} p<0.01\right), 1.10 \mathrm{E}+06$ of Dox $2.5 \mathrm{mg} / \mathrm{kg}$ group $\left({ }^{*} p<0.05\right)$, $1.36 \mathrm{E}+05$ of Rapa plus Dox $5 \mathrm{mg} / \mathrm{kg}$ (**** $p<0.0001$ ) and $5.13 \mathrm{E}+05$ of Rapa plus Dox $2.5 \mathrm{mg} / \mathrm{kg}$ group $(* * *<0.001)$. At day +42 , two animals from the control group died before BLI acquisition.

The tumor volume inhibition, determined by the various treatments, indicated that Rapa and Dox, alone or in combination, showed a significant effect against GBM growth (Figure 4A). 
A
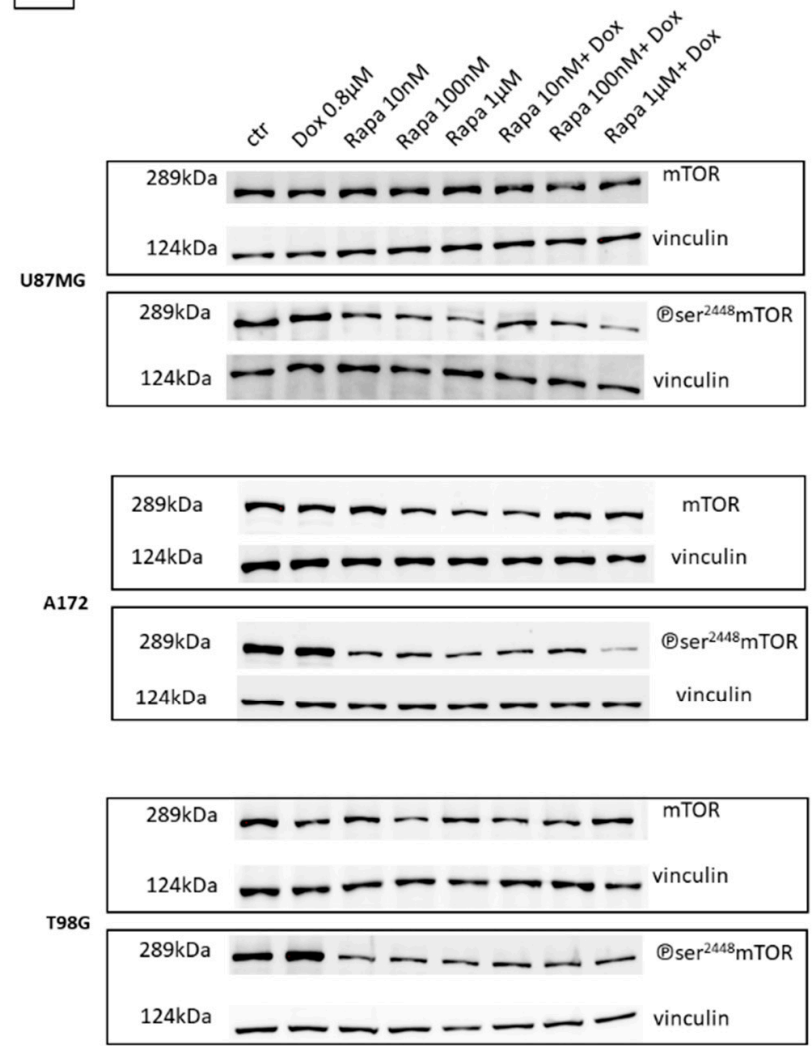

B
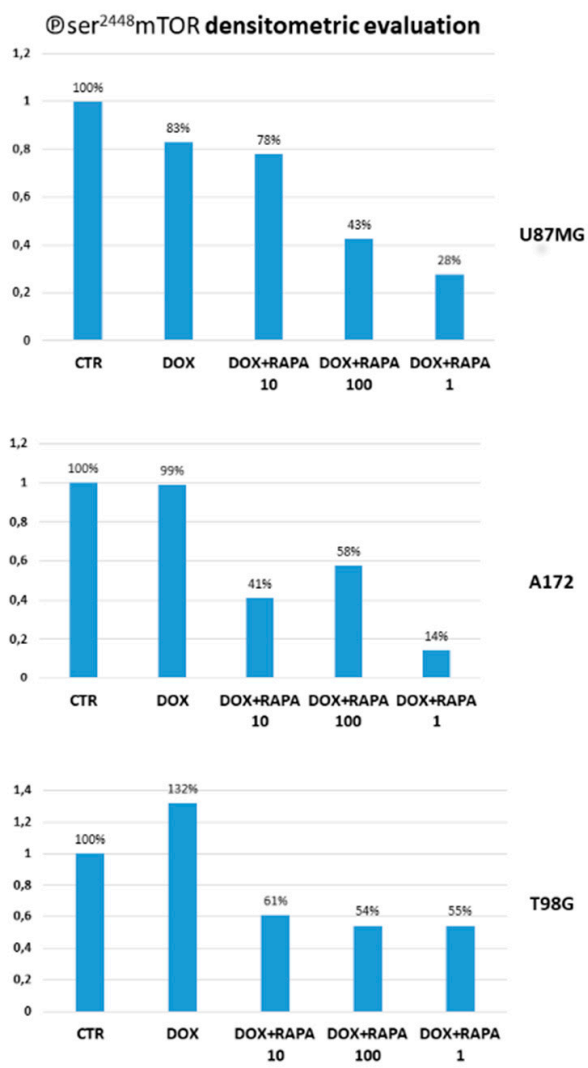

Figure 3. Effect of Rapa on mTOR S2448 phosphorylation (A) Immunoblot analysis of mTOR and ${ }^{\circledR} \operatorname{ser}^{2448}$ mTOR signaling in U87MG, A172, and T98G glioma cells, treated with Dox, various concentrations of Rapa and their combinations for $8 \mathrm{~h}$. Vinculin was used as a protein loading control; (B) Densitometric analysis of ${ }^{\circledR} \operatorname{ser}^{2448}$ mTOR signaling in U87MG, A172, and T98G glioma cells. All samples were normalized vs. vinculin and ${ }^{\circledR} \mathrm{ser}^{2448} \mathrm{mTOR}$ levels were referred to CTR $(100 \%)$.

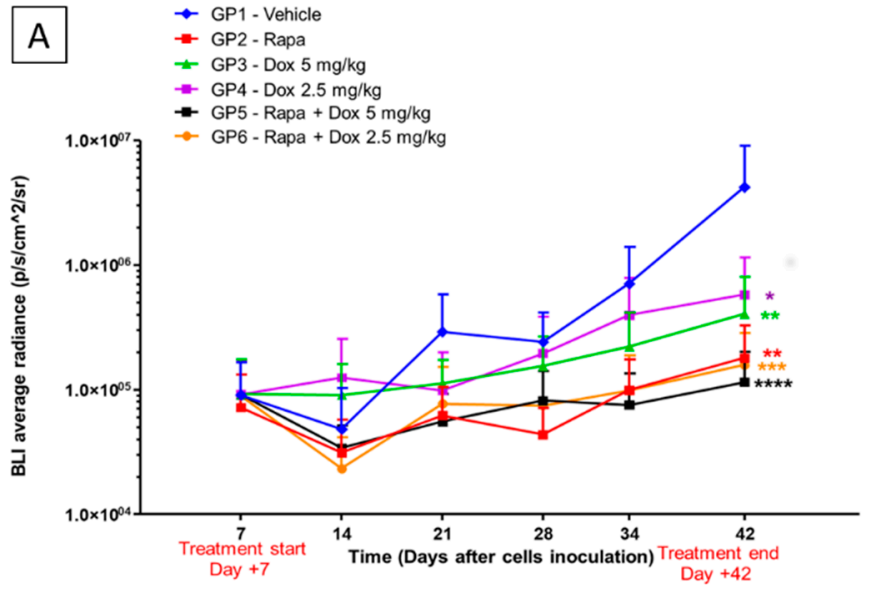

Figure 4. Cont.

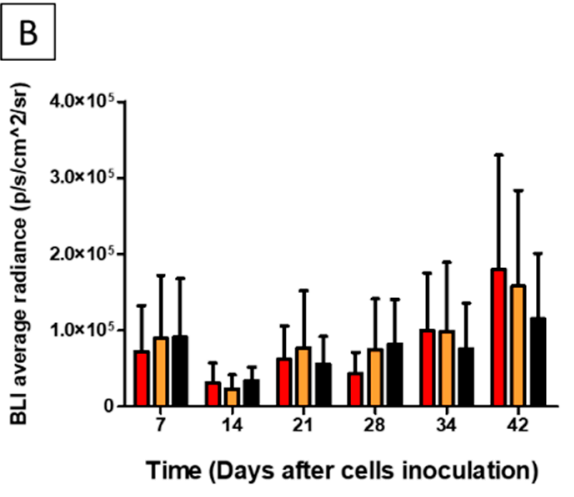



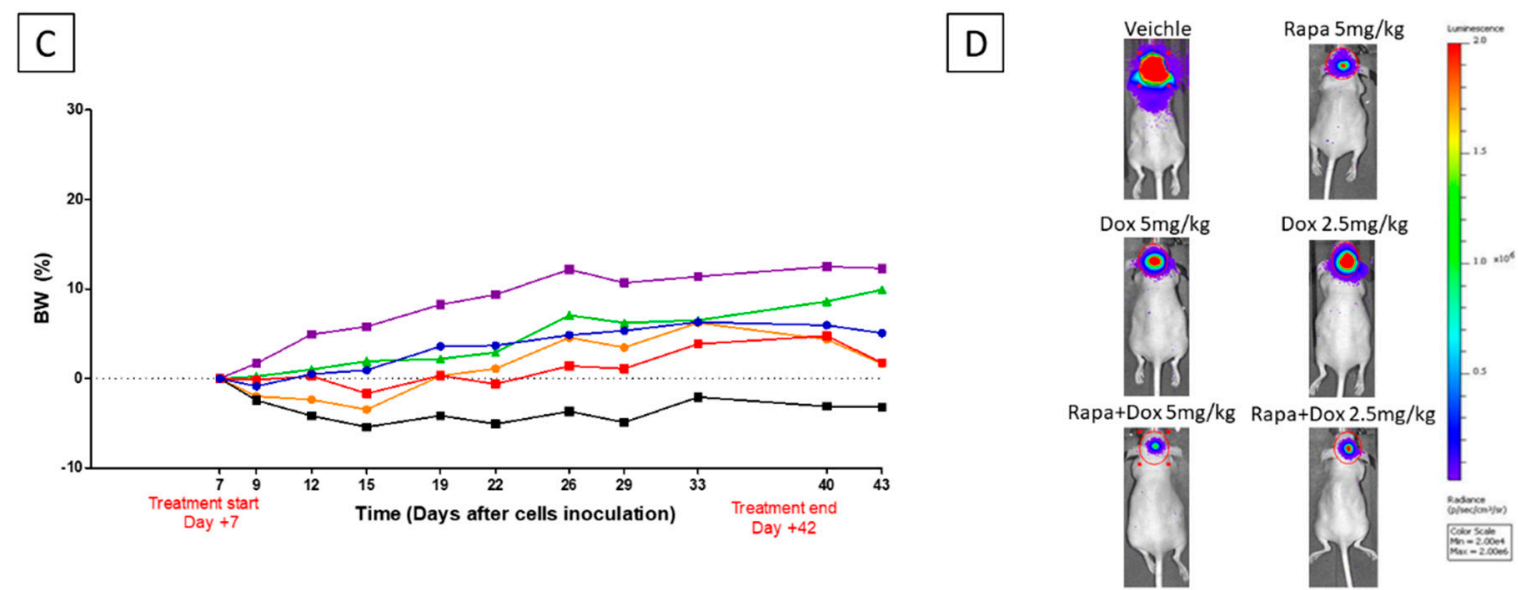

Figure 4. Bioluminescence signals throughout the experimental period. (A) Comparison of BLI acquisitions between all experimental groups. Outliers were removed by using the ROUT test $(\mathrm{Q}=1 \%)$. Statistical analysis was performed by Mann-Whitney U-test at day +42 (end of treatment) $\left({ }^{*} p<0.05\right.$; ** $p<0.01{ }^{* * *} p<0.001 ;{ }^{* * * *} p<0.0001$ vs. Vehicle-treated group); (B) BLI trends in GP2, GP5, and GP6 throughout the experimental time; (C) Percent of body weight variation throughout the experimental period; (D) Representative images of BLI acquisition of each groups at day +42 (end of treatment).

All treatments determined a tumor growth inhibition from $86 \%$ to $97 \%$ but the most significant result has been given by Rapa plus Dox $5 \mathrm{mg} / \mathrm{kg}$ combination $(-97.29 \%$ ). The in vivo results also revealed that the combination with Dox slight sensitized GBM to the cytotoxic effect of Rapa (Rapa $-95.76 \%$, Rapa plus Dox $2.5 \mathrm{mg} / \mathrm{kg}-96.28 \%$, Rapa plus Dox $5 \mathrm{mg} / \mathrm{kg}-97.29 \%$ ). Moreover, in accordance with in vitro results, Figure 4B shows BLI trends of GP2, GP5 and GP6 during the experimental time. In this figure, is possible to appreciate the total inversion of BLI value that occurs between these groups, underling the concept that even if Rapa alone appear to be effective against GBM its effect is maximal in the first stage, while prolonging treatments, the effectiveness of Rapa decreased and combined treatments showed a higher BLI reduction.

Side effects of the treatments, evaluated as BW\% variations of treated groups compared to control group, are reported in Figure 4C.

At day +43 , we reported a BW\% variation of $1.75 \%$ in Rapa group, $9.91 \%$ in Dox $5 \mathrm{mg} / \mathrm{kg}$ group, $12.32 \%$ in Dox $2.5 \mathrm{mg} / \mathrm{kg}$ group, $-3.19 \%$ in Rapa plus Dox $5 \mathrm{mg} / \mathrm{kg}$ group and $1.64 \%$ in Rapa plus Dox $2.5 \mathrm{mg} / \mathrm{kg}$ group.

This data indicated that, even if Rapa plus Dox $5 \mathrm{mg} / \mathrm{kg}$ treatment shows the highest effect on tumor growth inhibition (Figure 4D), this combination was the only one that caused weight loss (with a negative $\mathrm{BW} \%$ variation), resulting in greater toxicity.

\subsection{Proliferation Index -Ki67 Quantitative Analysis}

At the end of the treatments (day +42$)$ brains of five animals of each group were collected to undergo histological evaluation.

Samples were explored at different cutting levels and serial slides were stained with haematoxylin-eosin or Ki67 immunostaining, in order to calculate the proliferation index within the tumor mass.

As reported in Figure 5A,B, Rapa monotherapy induced a meaningful and statistically significant decrease in the proliferation index compared to control samples, while Dox ( 5 and $2.5 \mathrm{mg} / \mathrm{kg}$ ) induced a slight and not significant reduction of the value in monotherapy regimen.

Combined treatments showed better results than monotherapies. In particular, Rapa plus Dox 2.5 $\mathrm{mg} / \mathrm{kg}$ samples present a proliferation index comparable to Rapa treatment while, Rapa plus Dox 5 $\mathrm{mg} / \mathrm{kg}$ result the best treatment with the lowest value of proliferation and a statistically significant difference compared to control ( $8.92 \pm 3.3$ vs. $\left.31.87 \pm 12.98 ;{ }^{*} p<0.05\right)$ (Figure $5 B$ ). 
A

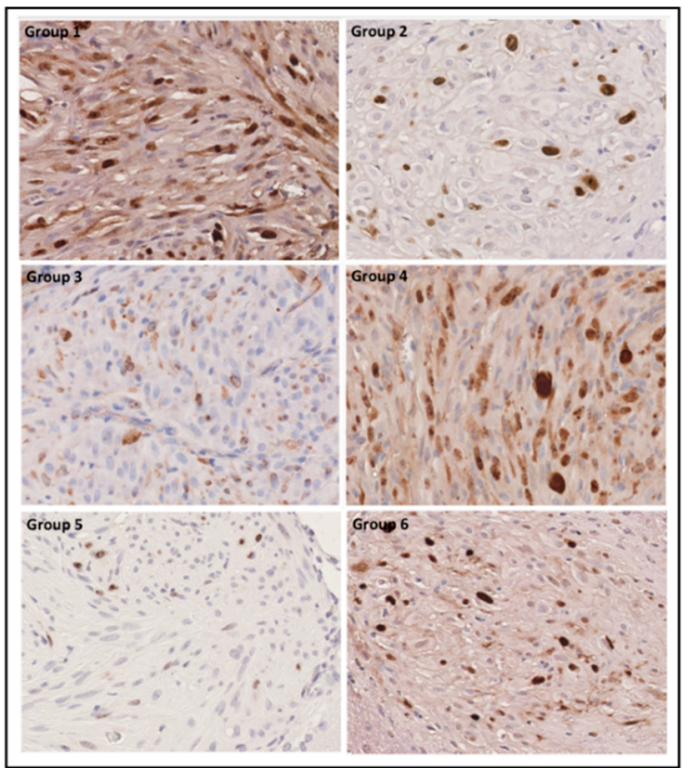

B

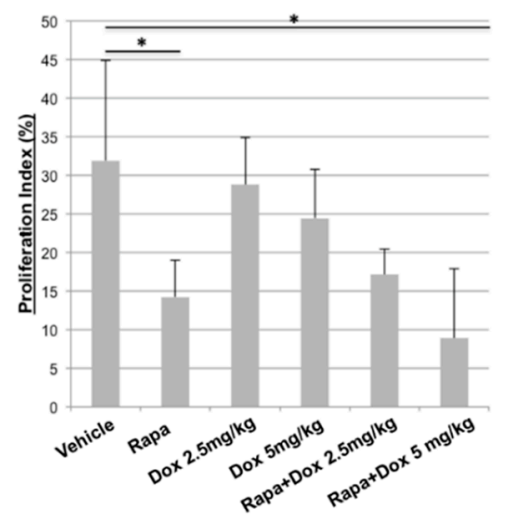

Figure 5. Immunohistochemical evaluation of therapies on glioma proliferation (A) Representative images of Ki67-immunoreactivity showed by tumor samples from each groups. Formalin-fixed, Wax-embedded, anti-Ki67 IHC stained slides-Final Magnification 400×; (B) Proliferation index comparison between vehicle group vs. treated groups; statistical analysis was performed through the unpaired Mann-Whitney U-test and ANOVA with Tukey correction $\left({ }^{*} p<0.05\right)$.

\section{Discussion}

The role of chemotherapy for the treatment of GBM patients remains an ongoing challenge for the scientific community.

Numerous drugs have shown great toxicity in GBM in vitro models, but clinical trials, using consolidated and new agents, have showed no significant improvement in terms of overall survival and progression-free survival [12]. These disappointing results can, at least in part, be explained by (i) the inability to deliver more effective anticancer agents, as Dox, into the CNS across the BBB, avoiding various resistance mechanisms, including the active efflux of anticancer drugs mediated by ATP-binding cassette (ABC) transporters as P-gp [13]; (ii) the acquisition of adaptive and resistance mechanisms of GBM cells in the presence of prolonged treatment with metabolic activity modulators, such as Rapa [14].

It is becoming clear that combination or sequential treatment modalities, that target multiple pathways, can lead to better control of aberrant cell proliferation.

We have investigated the potential of a new pharmacological approach, combining Dox, an anthracycline more effective than TMZ against GBM [4,15] and Rapa, a potent inhibitor of mTOR that is largely involved in GBM proliferation and progression [16].

In accordance with literature data [17], our in vitro results demonstrated the cytotoxic effect of Rapa on GBM cell lines with different degrees of sensibility. At $24 \mathrm{~h}$ of treatment, the cytotoxic effect can be attributed exclusively to Rapa; significance was obtained only in treatments including Rapa vs. controls. In the same cell lines, excluding T98G (that we have previously reported to be resistant to Dox [5]), Dox effectiveness was appreciable only $48 \mathrm{~h}$ after treatments. This data indicated that Rapa blocks cells proliferation in a short period while Dox cytotoxic mechanism requires more time to induce GBM cell death. An interesting result was obtained in T98G cells, prolonging treatments at $72 \mathrm{~h}$.

In this case, T98G remained resistant to Dox but showed a high cytotoxic effect when co-treated with Dox and Rapa; the most interesting result was the significance achieved comparing Rapa vs. Rapa plus Dox groups, suggesting that Rapa probably sensitizes T98G cells to Dox. 
Moreover, we have investigated the mechanism underlying the increased cytotoxicity of the combined treatment Rapa plus Dox compared with only Dox focusing our attention on two goals: the effect of treatments on mTOR modulation and effect of treatments on Dox uptake.

mTOR is a critical mediator of numerous cellular signals in oncogenesis [8] and consists of two distinct components: mTORC1 and mTORC2. Rapa-sensitive mTORC1 directly targets ribosomal protein S6 kinase (p70S6K) and eukaryotic translation initiation factor 4E-binding protein 1 (4E-BP1) to promote cap-dependent protein translation $[18,19]$. At molecular level, Ser2448 is the most studied phosphorylation sites on mTORC1. Recent studies have demonstrated that S2448 phosphorylation is a biomarker of mTORC1 activity [20].

We reported a clear modulation of the combined treatments on S2448 phosphorylation, vs. control and Dox treatment, in GBM cell lines (Figure 3B). The effect was more appreciable in U87MG and A172, Dox sensible cells, with a maximum phosphorylation inhibition associated with Dox plus Rapa 1 $\mu \mathrm{M}$ co-treatment ( $72 \%$ and $86 \%$ vs. control).

T98G, a Dox resistant cell line, showed a minor modulation with a 50\% S2448 phosphorylation inhibition. This 50\% mTOR conserved activity in association with Dox resistance can be identified as contributing causes of T98G multidrug resistance (MDR) phenotype. These interesting results, in association with cytotoxic data, clearly show that MDR is a complex phenotype, mediated from different factors and pathways. Combinational or sequential treatment modalities that investigate multiple targets can lead to better control of aberrant cell proliferation.

Interestingly, Arcella et al. also found that, despite producing mTOR inhibition, Rapa administration did not produce a compensatory increase in Akt levels. In detail, they found that Rapa robustly decreased phosphorylated (p)Akt levels while non-phosphorylated Akt levels were steady, producing a decrease in the ratio between pAkt and Akt. These data were consistent along patients and U87MG cell line [14].

Literature data about the interaction between Rapa and P-gp, the major responsible of drug efflux at BBB level, are currently conflicting.

Different studies reported that Rapa and its analogue, Tacrolimus, tested at $2.5 \mu \mathrm{M}$ concentration, were able to modulate P-gp and BCRP activity [21,22].

On the other hand, in vitro data on fibrosarcoma, leukaemia, and immune cells reported that Rapa did not modulate P-gp, MRP-1, or BCRP at its clinically achievable concentration of $0.25 \mu \mathrm{M}$. The same result was also obtained using a Rapa analogue, Tacrolimus, at its clinically achievable concentration of $0.08 \mu \mathrm{M}$.

In accordance with these outcomes, we also report comparable data evaluating the effect of Rapa on Dox uptake by using an in vitro model of BBB. Dox is a P-gp substrate and, taking advantage of its autofluorescence, we have quantified Dox intracellular accumulation in presence of various doses of Rapa in MDCKII P-gp transfected cells. As reported in Figure 3B, no differences in Dox signal were observed comparing all treated samples vs. control and neither vs. Dox only. This result indirectly demonstrated that Rapa (at tested concentrations) has no effect on Dox P-gp-mediate transport across BBB (Figure 2B).

These results indicated that the affinity and modulatory effect of Rapa, on the $A B C$ transporter, was strongly related to the used dosage of the mTOR inhibitor.

Considering the interesting in vivo results obtained by Arcella et al., we have tested the efficacy of the co-treatment Rapa plus Dox in a xenograft mice model of human GBM. They reported a strong efficacy of Rapa ( $5 \mathrm{mg} / \mathrm{kg}$ ) on cell proliferation, tumor volume inhibition, and survival. Therefore, we tested the same dose for our study as well. [14]. Dox doses, for in vivo experiments, have been replaced from our previous experiments [6].

At the end of the experiment, day +42 , our results indicated that all treatments had noteworthy effects on BLI and tumor volume inhibitions, with different degrees of significance (Figure 4A). The most effective approaches resulted, for both treatments, Rapa plus Dox $5 \mathrm{mg} / \mathrm{kg}$ and Rapa plus Dox $2.5 \mathrm{mg} / \mathrm{kg}$. These results were also supported by immunohistological analysis. Nuclear Ki-67 
immunopositivity is higher in rapidly growing malignant tumors than in their slowly growing benign counterparts [23-25].

Five brains for each group undergoes to histological evaluation. The difference in the number of the examined samples is due to the difficult interception of the tumor mass, especially in the co-treated groups. Our samples were stained with haematoxylin-eosin or Ki67 immunostaining, in order to calculate the proliferation index within the tumor mass (Figure 5A). Rapa plus Dox $2.5 \mathrm{mg} / \mathrm{kg}$ samples presented a proliferation index comparable to Rapa treatment while, Rapa plus Dox $5 \mathrm{mg} / \mathrm{kg}$ result in the best treatment with the lowest value of proliferation and a statistically significant difference compared to control.

Rapa plus Dox $5 \mathrm{mg} / \mathrm{kg}$ resulted in the best treatment under different points of view, but it was associated with a greater toxicity, evaluated as BW\% variation. Interestingly, even if Rapa had a very slight effect on Dox uptake (Figure 2), the combination Rapa plus Dox $2.5 \mathrm{mg} / \mathrm{kg}$ showed a tumor inhibition like Rapa plus Dox $5 \mathrm{mg} / \mathrm{kg}$ combination. It is likely that in the presence of Rapa adjuvant effects, the Dox result is able to express its cytotoxic potential at lower concentrations, with reduced side effects.

In conclusion, our results demonstrate that Rapa plus Dox treatment had the advantage (i) to reduce tumor cells proliferation by reducing the mTOR activation; (ii) to improve the cytotoxic effect of Dox; and (iii) to reduce the risk for a clinically relevant negative impact of protective effects of mTOR modulation, combining the antiproliferative/cytotoxic effect of Rapa with the cytotoxic effect of Dox (iiii) to make possible a reduction of the anthracycline dosage and consequently, Dox-mediated side effects of the treatments.

Further studies are necessary to better understand the potentiality of the combined treatment Rapa plus Dox, but this safe and non-invasive approach could be a successful strategy in the treatment of aggressive tumors as GBM.

Author Contributions: A.L.I. performed cell cultures, in vitro assays, statistical analysis, and drafted the manuscript. M.D.R. collaborated in the planning of the experiments, analysis, and evaluation of the results. C.P. processed the murine model. M.d.M. and L.G. collaborated in the evaluations of the results, literature search, and writing of reports and papers. I.S. conceived the study and supervised the project.

Funding: This research was funded by Fondazione Ospedale Pediatrico Meyer, Florence, Italy.

Acknowledgments: We are grateful to Francesco Cardile, BIOGEM Research Institute, Italy, Monica Muratori and Elisabetta Baldi, University of Florence, Italy, for their skillful technical assistance and to J.H.M Schellens, Netherlands Cancer Institute, Amsterdam, for generously providing MDCKII cell lines.

Conflicts of Interest: The authors declare no conflict of interest.

\section{References}

1. Stupp, R.; Mason, W.P.; van den Bent, M.J.; Weller, M.; Fisher, B.; Taphoorn, M.J.; Belanger, K.; Brandes, A.A.; Marosi, C.; Bogdahn, U.; et al. Radiotherapy plus concomitant and adjuvant temozolomide for glioblastoma. N. Engl. J. Med. 2005, 352, 987-996. [CrossRef] [PubMed]

2. Cloughesy, T.F.; Cavenee, W.K.; Mischel, P.S. Glioblastoma: From molecular pathology to targeted treatment. Annu. Rev. Pathol. 2014, 9, 1-25. [CrossRef] [PubMed]

3. Brennan, C.W.; Verhaak, R.G.; McKenna, A.; Campos, B.; Noushmehr, H.; Salama, S.R.; Zheng, S.; Chakravarty, D.; Sanborn, J.Z.; Berman, S.H.; et al. The somatic genomic landscape of glioblastoma. Cell 2013, 155, 462-477. [CrossRef] [PubMed]

4. Veringa, S.J.; Biesmans, D.; van Vuurden, D.G.; Jansen, M.H.; Wedekind, L.E.; Horsman, I.; Wesseling, P.; Vandertop, W.P.; Noske, D.P.; Kaspers, G.J.; et al. In vitro drug response and efflux transporters associated with drug resistance in pediatric high grade glioma and diffuse intrinsic pontine glioma. PLoS ONE 2013, 8, e61512. [CrossRef] [PubMed]

5. Sardi, I.; Fantappiè, O.; la Marca, G.; Giovannini, M.G.; Iorio, A.L.; da Ros, M.; Malvagia, S.; Cardellicchio, S.; Giunti, L.; de Martino, M.; et al. Delivery of doxorubicin across the blood-brain barrier by ondansetron pretreatment: A study in vitro and in vivo. Cancer Lett. 2014, 353, 242-247. [CrossRef] [PubMed] 
6. da Ros, M.; Iorio, A.L.; Consolante, D.; Cardile, F.; Muratori, M.; Fantappiè, O.; Lucchesi, M.; Guidi, M.; Pisano, C.; Sardi, I. Morphine modulates doxorubicin uptake and improves efficacy of chemotherapy in an intracranial xenograft model of human glioblastoma. Am. J. Cancer Res. 2016, 6, 639-648. [PubMed]

7. Dowling, R.J.; Topisirovic, I.; Fonseca, B.D.; Sonenberg, N. Dissecting the role of mTOR: Lessons from mTOR inhibitors. Biochim. Biophys. Acta 2010, 1804, 433-439. [CrossRef]

8. Sami, A.; Karsy, M. Targeting the PI3K/AKT/mTOR signaling pathway in glioblastoma: Novel therapeutic agents and advances in understanding. Tumour Biol. 2013, 34, 1991-2002. [CrossRef]

9. Guertin, D.A.; Sabatini, D.M. Defining the role of mTOR in cancer. Cancer Cell 2007, 12, 9-22. [CrossRef]

10. Chiang, G.G.; Abraham, R.T. Phosphorylation of mammalian target of rapamycin (mTOR) at Ser-2448 is mediated by p70S6 kinase. J. Biol. Chem. 2005, 280, 25485-25490. [CrossRef]

11. Heimberger, A.B.; Wang, E.; McGary, E.C.; Hess, K.R.; Henry, V.K.; Shono, T.; Cohen, Z.; Gumin, J.; Sawaya, R.; Conrad, C.A.; et al. Mechanisms of action of rapamycin in gliomas. Neuro Oncol. 2005, 7, 1-11. [CrossRef] [PubMed]

12. Omuro, A.M.; Faivre, S.; Raymond, E. Lessons learned in the development of targeted therapy for malignant gliomas. Mol. Cancer Ther. 2007, 6, 1909-1919. [CrossRef] [PubMed]

13. Wijaya, J.; Fukuda, Y.; Schuetz, J.D. Obstacles to Brain Tumor Therapy: Key ABC Transporters. Int. J. Mol. Sci. 2017, 18. [CrossRef] [PubMed]

14. Ronellenfitsch, M.W.; Brucker, D.P.; Burger, M.C.; Wolking, S.; Tritschler, F.; Rieger, J.; Wick, W.; Weller, M.; Steinbach, J.P. Antagonism of the mammalian target of rapamycin selectively mediates metabolic effects of epidermal growth factor receptor inhibition and protects human malignant glioma cells from hypoxia-induced cell death. Brain 2009, 132, 1509-1522. [CrossRef] [PubMed]

15. Ryskalin, L.; Lazzeri, G.; Flaibani, M.; Biagioni, F.; Gambardella, S.; Frati, A.; Fornai, F. mTOR-Dependent Cell Proliferation in the Brain. Biomed. Res. Int. 2017, 2017, 7082696. [CrossRef] [PubMed]

16. Duzgun, Z.; Eroglu, Z.; Biray Avci, C. Role of mTOR in glioblastoma. Gene 2016, 575, 187-190. [CrossRef] [PubMed]

17. Arcella, A.; Biagioni, F.; Antonietta Oliva, M.; Bucci, D.; Frati, A.; Esposito, V.; Cantore, G.; Giangaspero, F.; Fornai, F. Rapamycin inhibits the growth of glioblastoma. Brain Res. 2013, 1495, 37-51. [CrossRef] [PubMed]

18. Tamburini, J.; Green, A.S.; Bardet, V.; Chapuis, N.; Park, S.; Willems, L.; Uzunov, M.; Ifrah, N.; Dreyfus, F.; Lacombe, C.; et al. Protein synthesis is resistant to rapamycin and constitutes a promising therapeutic target in acute myeloid leukemia. Blood 2009, 114, 1618-1627. [CrossRef]

19. Gingras, A.C.; Gygi, S.P.; Raught, B.; Polakiewicz, R.D.; Abraham, R.T.; Hoekstra, M.F.; Aebersold, R.; Sonenberg, N. Regulation of 4E-BP1 phosphorylation: A novel two-step mechanism. Genes Dev. 1999, 13, 1422-1437. [CrossRef]

20. Copp, J.; Manning, G.; Hunter, T. TORC-specific phosphorylation of mammalian target of rapamycin (mTOR): Phospho-Ser2481 is a marker for intact mTOR signaling complex 2. Cancer Res. 2009, 69, 1821-1827. [CrossRef]

21. Arceci, R.J.; Stieglitz, K.; Bierer, B.E. Immunosuppressants FK506 and rapamycin function as reversal agents of the multidrug resistance phenotype. Blood 1992, 80, 1528-1536. [PubMed]

22. Gupta, A.; Dai, Y.; Vethanayagam, R.R.; Hebert, M.F.; Thummel, K.E.; Unadkat, J.D.; Ross, D.D.; Mao, Q. Cyclosporin A, tacrolimus and sirolimus are potent inhibitors of the human breast cancer resistance protein (ABCG2) and reverse resistance to mitoxantrone and topotecan. Cancer Chemother. Pharmacol. 2006, 58, 374-383. [CrossRef] [PubMed]

23. Tüttenberg, J.; Fink, W.; Back, W.; Wenz, F.; Schadendorf, D.; Thomé, C. A rare primary sellar melanoma. Case report. J. Neurosurg. 2004, 100, 931-934. [CrossRef] [PubMed]

24. Rousseau, A.; Kujas, M.; van Effenterre, R.; Boch, A.L.; Carpentier, A.; Leroy, J.P.; Poirier, J. Primary intracranial myopericytoma: Report of three cases and review of the literature. Neuropathol. Appl. Neurobiol. 2005, 31, 641-648. [CrossRef] [PubMed]

25. Ohsie, S.J.; Sarantopoulos, G.P.; Cochran, A.J.; Binder, S.W. Immunohistochemical characteristics of melanoma. J. Cutan Pathol. 2008, 35, 433-444. [CrossRef] [PubMed]

(C) 2019 by the authors. Licensee MDPI, Basel, Switzerland. This article is an open access article distributed under the terms and conditions of the Creative Commons Attribution (CC BY) license (http:/ / creativecommons.org/licenses/by/4.0/). 